\title{
Improving the Energy Efficiency of Two-Tier Heterogeneous Cellular Networks through Partial Spectrum Reuse
}

\author{
Dongxu Cao, Student Member, IEEE, Sheng Zhou, Member, IEEE, and Zhisheng Niu, Fellow, IEEE
}

\begin{abstract}
Partial Spectrum Reuse (PSR) in the second tier of two-tier heterogeneous cellular networks has a potential to improve spectrum efficiency by reducing inter-cell interference, and thus energy efficiency as well by deploying less or switching off more Base Stations (BSs). In this paper, we analyze the optimal PSR factor, defined as the portion of spectrum reused by micro cells in two-tier heterogeneous networks, which is not in an explicit form generally. Then, a closed-form limit of the optimal PSR factor is derived as the ratio of the user rate requirement over the whole system spectrum bandwidth is approaching zero, based on which a threshold of the micro-BS energy cost is also derived to determine which type of BSs is preferable. Specifically, one should deploy more micro BSs or switch off more macro BSs if the micro-BS energy cost is lower than the threshold. Otherwise, the optimal choice is the opposite. This threshold with the PSR scheme is higher than that without PSR scheme, i.e., PSR can improve both spectrum efficiency and energy efficiency. Numerical results show that adopting PSR can reduce the network energy consumption by up to $50 \%$ when the transmit power of macro BSs is $10 \mathrm{~dB}$ higher than that of micro BSs.
\end{abstract}

Index Terms-Heterogeneous cellular networks, partial spectrum reuse, poisson point process, energy efficiency.

\section{INTRODUCTION}

$\mathbf{T}$ ODAY'S cellular networks are the major sources of energy consumption in ICT industry. Taking China as an example, there had been over 1.0 million Base Stations (BSs) deployed by the three major operators consuming as much as 28.9 billion KWh electric power a year by 2009 [1]. As the service demand increases, the energy consumption will also increase in the future. Thus, energy-efficient approaches are urgently required by network vendors. On the other hand, the multi-tier heterogeneous cellular network composed of macro, outdoor pico and indoor femto BSs is expected as a promising solution of the future $4 \mathrm{G}$ cellular system to provide high quality of service (QoS) [2]-[4]. In this sense, how to plan and how to operate heterogeneous cellular networks in an energy-efficient way are important issues.

Manuscript received November 9, 2012; revised January 31, 2013; accepted April 9, 2013. The associate editor coordinating the review of this paper and approving it for publication was T. J. Lim.

The authors are with Tsinghua National Laboratory for Information Science and Technology, Dept. of Electronic Engineering, Tsinghua University, Beijing 100084, China (e-mail: cdx08@mails.tsinghua.edu.cn, \{sheng.zhou, niuzhs\}@ @tsinghua.edu.cn).

This work is sponsored in part by the National Basic Research Program of China (2012CB316001), the Nature Science Foundation of China (61201191, 60925002, 61021001), and Hitachi Ltd.

Digital Object Identifier 10.1109/TWC.2013.052213.121751
In this paper, we focus on two-tier heterogeneous networks, and the BSs in the second tier are abstracted into "micro" BSs. As the BSs are the major energy consumers of cellular networks [13], an effective way to improve the energy efficiency is to optimize the combination of macro and micro BS densities, i.e., to deploy or switch off macro and micro BSs with proper numbers. However, to achieve this, it is necessary to make full use of system spectrum in heterogeneous networks.

Our previous work [5] assumes that the universal spectrum reuse is adopted, in which each BS, either the macro or the micro, occupies the whole system spectrum. However, it will bring severe inter-cell interference, and thus limit the QoS performance of macro cells, for the number of users in macro cells is generally much larger than that in micro cells. Therefore, a spectrum reuse scheme that micro BSs partially reuse the system spectrum, namely Partial Spectrum Reuse (PSR) [6], can reduce the intensity of interfering micro BSs, and thus reduce the inter-cell interference generated from them. With the proper design of PSR scheme, the QoS performance, e.g., the service outage performance considered in this paper, can be improved. Further to meet the same QoS requirement, less macro and micro BSs are required, and in such sense, the network energy consumption is reduced.

Although PSR schemes can be realized through a preplanned fixed pattern, an adaptive and distributed way via dynamic bandwidth allocation is preferable because the large amount of unplanned micro BSs [3] make the centralized frequency planning difficult [7]. In this paper, we consider a distributed PSR scheme, called " $\beta$-PSR", in which each macro BS can allocate the whole spectrum, but each micro BS can only allocate a portion $\beta$ ( $\beta \in(0,1]$, PSR factor) of the whole spectrum. Further, in each micro BS, the $\beta$-portion of spectrum is generated randomly and independently, which is easy to realize as it requires little information exchange.

Then two questions arise: what is the optimal value of the PSR factor $\beta^{*}$ given system parameters such as BS density and user intensity? Furthermore, how many macro and micro BSs should be deployed or switched off to minimize network energy consumption in heterogeneous networks? In this paper, we extend our previous work [5] with PSR schemes to answer these two problems. The answer to the first question can show how to adjust PSR schemes according to the traffic intensity, the macro/micro BS density, and their transmit power, while the answer to the second question can reveal the best combination of BSs with the associated PSR scheme 
to minimize the network energy consumption.

\section{A. Related Work}

Frequency reuse is a classic topic in cellular networks, varying from the classic reuse- $n$ ( $n=3,7$ typically) schemes to the current universal frequency reuse in the CDMA-based 3G cellular networks. Similarly, Fractional Frequency Reuse schemes [8]-[10] based on users' geographical information were proposed. However, these frequency reuse schemes are mainly designed for homogeneous cellular networks, and cannot be directly applied to heterogeneous networks. Among the very limited work on spectrum reuse in heterogeneous networks, Ref. [6] showed the feasibility of femtocells partially reusing GSM spectrum, which is similar to our PSR schemes. We extend the work in [6] to the optimal PSR factor design, and investigate the impact of PSR schemes on the energy efficiency performance of heterogeneous networks.

Recently, the energy saving in cellular networks has become increasingly important. Accordingly, dynamic operation control schemes to optimize energy efficiency in cellular networks have been proposed [11]-[13], mainly focusing on dynamic BS sleeping. There are also some works [14]-[17] showing the benefits of deploying low power micro BS sites to improve energy efficiency through system-level simulations. Nevertheless, few consider dynamic BS sleeping jointly with spectrum reuse in heterogeneous cellular networks.

Current researches about cellular network planning mainly focus on the practical deployment algorithm design [18]-[22]. Mostly, it is an NP-hard mixed integer programming problem with a lot of system parameters and constraints, and thus many numerical solutions have been proposed. However, each solution is problem-specific and can be viewed as subsequent steps of our BS density problem. Among the very limited work on optimal BS density, Ref. [23] focused on the maximal inter-BS distance for CDMA cellular networks based on the hexagonal cellular network model. However, this work cannot be directly applied to capacity extension or dynamic BS sleeping.

In this paper, we assume the location of BSs, either the macro or the micro, follows a random point process, PPP (Poisson Point Process) [24]-[26], and the cell topology is determined as the weighted-Voronoi tessellation [5]. Particularly, this paper is mainly based on our previous work [5]. In Ref. [5], we showed the best combination of macro and micro BS densities to be deployed or switched off in heterogeneous cellular networks, but based on the universal spectrum reuse scheme. This paper extends Ref. [5] with PSR schemes. Besides, Ref. [25] is also particularly related, as it derived the coverage probability with spectrum reuse in the cellular network model using stochastic geometry.

\section{B. Contributions and Organization}

In this paper, we design a distributed PSR scheme, and investigate its impact on capacity extension and dynamic BS sleeping to improve energy efficiency in heterogeneous networks. The user QoS metric is measured as the service outage probability that the instantaneous service rate of a user is lower than a predefined value. To meet the QoS requirement, the service outage probability should be less than a threshold.
We present an asymptotic analysis of the QoS metric, based on which a closed-form limit of the optimal PSR factor is derived. This limit is an explicit function of traffic intensity, the macro/micro BS density and their transmit power, when the ratio of the user rate requirement over the system bandwidth is approaching zero. Furthermore, our numerical results show that for most current rate requirements, the optimal PSR factor is well approximated by this limit, which motivates a distributed PSR scheme design with near-optimal performance.

As the BS density is an important factor for network design, we consider the optimal BS density problem jointly with PSR schemes. We show that generally the network energy cost is a quasi-concave function, and thus there exists a threshold of the micro-BS energy cost to determine which type of BSs is preferable. It is found that if the micro-BS energy cost is below this threshold, the optimal strategy is to deploy more micro BSs for capacity extension or to switch off more macro BSs for energy saving; otherwise, the optimal choice is the opposite. This threshold with the PSR scheme is definitely higher than that without PSR scheme, and the gap between them is the benefit that micro BSs get from PSR. Our numerical results also show that the PSR scheme can reduce up to $50 \%$ of network energy consumption when the transmit power of micro BSs is $10 \mathrm{~dB}$ lower than that of macro BSs, compared with no PSR schemes.

The rest of this paper is organized as follows: In Section II, the heterogeneous cellular system models are presented. Then the optimal PSR scheme is derived in Section III, after which the optimal BS density problem in heterogeneous cellular networks with PSR schemes is investigated in Section IV. Finally, Section V concludes this paper.

\section{Heterogeneous Cellular System Model}

We consider a heterogeneous cellular network deployed with two different types of BSs: macro BSs with high transmit power and high energy cost, and micro BSs with low transmit power and low energy cost. Both the macro and the micro BSs are assumed to be located according to independent homogeneous PPPs (Poisson Point Process). There are several advantages for choosing this model: I) Though the PPP model is not an exact fit, it reasonably approximates the variability introduced by practical constraints of macro BS locations [28] and the potential unplanned deployment [3] of micro BSs. II) This model is suitable to analyze capacity extension and dynamic BS sleeping [5], as the superposition of two or more independent PPPs, and the independent thinning of a PPP are still PPPs.

Users are also located according to a homogeneous PPP, and each user is always associated with the BS with the greatest average received signal strength. Each BS allocates the wireless resources (e.g., time slots or wireless spectrum) equally to the associated users. Fig. 1 shows an example of a heterogenous cellular network topology, in which a user may not be associated with the nearest micro BS but a further macro BS, due to different transmit power.

Wireless channel gain is modeled as path-loss multiplied by the Rayleigh fading, and no power control scheme is assumed. The noise is ignored, because typical cellular networks are 


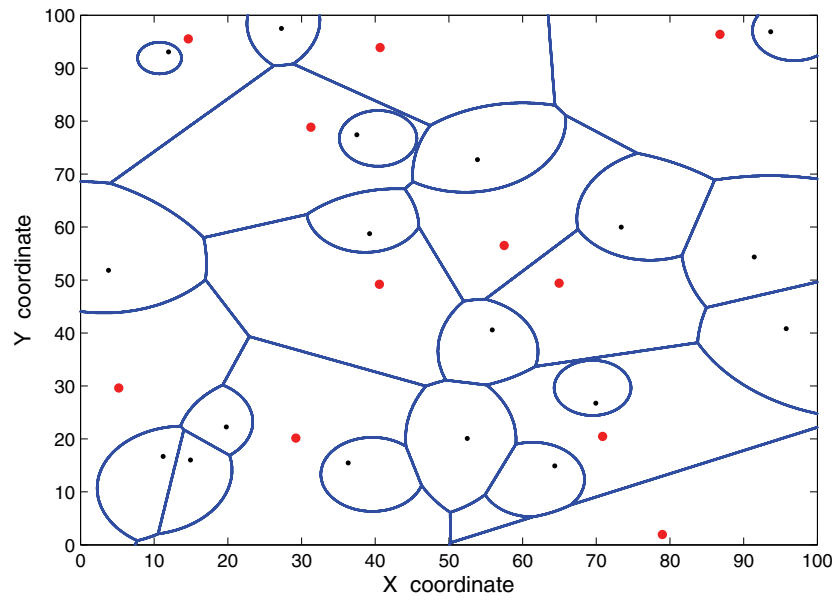

Fig. 1. An example of the heterogeneous cellular network model.

interference-limited [29], especially for downlink transmissions. We also assume that the $\beta$-PSR scheme is adopted, where each micro BS can only allocate a portion $\beta$ of the whole system spectrum $(\beta \in(0,1])$, and the $\beta$-portion of spectrum is generated randomly and independently. This $\beta$ PSR scheme, in addition to being analytically tractable through interference averaging, is easy to be realized, especially under the consideration of random deployment of micro BSs.

The QoS requirement is that the service outage probability of users in both macro and micro cells should be less than a threshold $\eta$. Note that the service outage occurs when the instantaneous service rate is lower than a predefined value $u$. Thus, the service outage probability is calculated as

$$
G=\mathbb{E}_{\{N, A\}}\left\{\operatorname{Pr}\left(\frac{W^{*}}{N} \log _{2}(1+\operatorname{SINR})<u \mid N, A\right)\right\},
$$

and similarly, the QoS requirement can be expressed as (2)

where $W^{*}$ is the bandwidth occupancy in a certain cell, and $N(N \geq 1)$ is the number of users within, whose distribution depends on the cell size $A$. The index $M$ denotes the macro and $m$ denotes the micro. For clarity, the mathematical symbols used in this paper are summarized in Table I.

Remark that generally, the user density and QoS requirement in macro cells are different from those in micro cells, shown as $\left(\lambda_{M}, \lambda_{m}\right)$ and $\left(u_{M}, u_{m}\right)$. However, as a baseline to find out the minimum cost among different BS density combinations, homogeneous user density and rate requirement are mainly considered in this paper. The general case with different user densities and rate requirements will be discussed for the PSR scheme design in Section III.

\section{Optimal Spectrum Reuse Factor}

For PSR, one would concern about what the optimal PSR factor $\beta^{*}$ should be to benefit both the macro-cell and microcell users. Intuitively, smaller $\beta$ brings lower inter-cell interference, but at the cost of less spectrum usage. For fairness, we set our target to minimize the maximum value of their service outage probabilities. This problem is then formulated as
TABLE I

SUMMARY OF THE NOTATIONS

\begin{tabular}{||c|c||}
\hline$\rho_{M}$ & macro BS density \\
\hline$\rho_{m}$ & micro BS density \\
\hline$C_{M}$ & energy consumption per macro BS \\
\hline$C_{m}$ & energy consumption per micro BS \\
\hline$P_{M}$ & transmit power of macro BS \\
\hline$P_{m}$ & transmit power of micro BS \\
\hline$\lambda$ & user intensity \\
\hline$u$ & downlink rate threshold \\
\hline$\eta$ & outage probability threshold \\
\hline$W$ & system spectrum bandwidth \\
\hline$\alpha$ & path loss exponent, valued in $(2,4]$ \\
\hline$\beta$ & PSR factor, valued in $(0,1]$ \\
\hline$c$ & $c \triangleq\left(\frac{P_{M}}{P_{m}}\right)^{\frac{2}{\alpha}}$ \\
\hline$\tau$ & $\tau \triangleq \frac{\rho_{m}}{\rho_{M}}$ \\
\hline$e$ & $e \triangleq \frac{C_{m}}{C_{M}}$ \\
\hline$\phi$ & $\phi \triangleq \frac{c \rho_{M}+\beta \rho_{m}}{c \rho_{M}+\rho_{m}} \frac{2}{\alpha-2}$ \\
\hline
\end{tabular}

$$
\begin{array}{ll}
\min _{\beta} & \max \left\{G_{M}(\beta), G_{m}(\beta)\right\} \\
\text { s.t. } & \beta \in(0,1],
\end{array}
$$

where $G_{M}(\beta)$ and $G_{m}(\beta)$ are defined in Eq. (2).

\section{A. QoS Metric Analysis}

To solve the above problem (3), we first need to calculate $G_{M}(\beta)$ and $G_{m}(\beta)$. Due to the similarity, only the calculation of $G_{M}(\beta)$ is presented. As shown in Ref. [5],

$$
\begin{aligned}
& \mathbb{E}_{\left\{N_{M}, A_{M}\right\}}\left\{\operatorname{Pr}\left(\frac{W}{N_{M}} \log _{2}(1+\mathrm{SINR})<u \mid N_{M}, A_{M}\right)\right\} \\
= & \mathbb{E}_{A_{M}} \mathbb{E}_{n \mid A_{M}}\left\{\operatorname{Pr}\left(\frac{W}{n+1} \log _{2}(1+\mathrm{SINR})<u \mid n, A_{M}\right)\right\} \\
= & \int_{0}^{\infty} \sum_{n=0}^{\infty} \operatorname{Pr}\left(\operatorname{SINR}<2^{\frac{(n+1) u}{W}}-1 \mid n, A\right) P_{A}(n) f_{M}(A) \mathrm{d} A
\end{aligned}
$$

where $f_{M}(A)$ represents the macro cell size distribution, and $P_{A}(n)$ denotes the remaining user number distribution, following the Poisson distribution as

$$
P_{A}(n)=\frac{(\lambda A)^{n}}{n !} \exp (-\lambda A) .
$$

The reason for " $n+1$ " in Eq. (4) is due to the Slivnyak's theorem [24] that if we condition on having a user within the macro cell, the number of remaining users follows the same distribution as if we do not have this condition, i.e., a Poisson distribution with parameter $\lambda A$.

As mentioned in [2], the typical transmit power of different types of BSs are: $5 \mathrm{~W}-40 \mathrm{~W}$ for the traditional macro, $250 \mathrm{~mW}$ $2 \mathrm{~W}$ for the outdoor pico, and less than $100 \mathrm{~mW}$ for the indoor femto. Therefore in [5], we studied a meaningful case in which the macro-BS transmit power is $10 \mathrm{~dB}$ higher 


$$
\begin{aligned}
& G_{M}=\mathbb{E}_{\left\{N_{M}, A_{M}\right\}}\left\{\operatorname{Pr}\left(\frac{W}{N_{M}} \log _{2}(1+\mathrm{SINR})<u \mid N_{M}, A_{M}\right)\right\}<\eta, \\
& G_{m}=\mathbb{E}_{\left\{N_{m}, A_{m}\right\}}\left\{\operatorname{Pr}\left(\frac{\beta W}{N_{m}} \log _{2}(1+\mathrm{SINR})<u \mid N_{m}, A_{m}\right)\right\}<\eta,
\end{aligned}
$$

than the micro-BS's, and the path loss exponent is 4, i.e., $c=\left(\frac{P_{M}}{P_{m}}\right)^{2 / \alpha}=3.1623$. We concluded that the macro and micro cell size distributions are accurately predicted by Gamma functions, as the following respectively,

$$
f_{M}(x)=x^{K_{M}-1} \frac{\exp \left(-\frac{c \rho_{M}+\rho_{m}}{c} K_{M} x\right)}{\left(\frac{c}{c \rho_{M}+\rho_{m}} \frac{1}{K_{M}}\right)^{K_{M}} \Gamma\left(K_{M}\right)},
$$

with $K_{M}=3.575 \frac{1+0.4106 \tau}{1+0.1673 \tau}=3.575 \frac{\rho_{M}+0.4106 \rho_{m}}{\rho_{M}+0.1673 \rho_{m}}$, and

$$
f_{m}(x)=x^{K_{m}-1} \frac{\exp \left(-\left(c \rho_{M}+\rho_{m}\right) K_{m} x\right)}{\left(\frac{1}{c \rho_{M}+\rho_{m}} \frac{1}{K_{m}}\right)^{K_{m}} \Gamma\left(K_{m}\right)}
$$

with $K_{m}=3.575 \frac{\tau+2.5327}{\tau+5.1952}=3.575 \frac{\rho_{m}+2.5327 \rho_{M}}{\rho_{m}+5.1952 \rho_{M}}$.

As the noise is ignored, the SINR of a random user is expressed as

$$
\mathrm{SINR}=\frac{h_{0} d_{0}^{-\alpha} P_{0}}{\sum_{i=1}^{\infty} \theta_{i} h_{i} d_{i}^{-\alpha} P_{i}},
$$

where the index 0 represents the home BS of this user, i.e., $d_{0}^{-\alpha} P_{0} \geq \max _{i=1,2, \ldots}\left\{d_{i}^{-\alpha} P_{i}\right\} ; h_{i}, d_{i}$ and $P_{i}$ respectively denote the fast fading factor, the distance and the transmit power of the $i$ th BS. Due to the defined PSR scheme, $\theta_{i}$ is used to denote whether the $i$ th BS transmits over the concerned spectrum or time slot. Specifically, if the $i$ th BS is a macro $\mathrm{BS}, \theta_{i}$ always equals to 1 ; otherwise, $\theta_{i}$ is a random variable, which equals to 1 with probability $\beta$, or else equals to 0 .

However, it is difficult to theoretically get the SINR distribution given cell size, because the cell size itself is difficult to calculate even for given BS deployment. Therefore, similarly with [27], we use the following approximation:

$$
\begin{aligned}
\operatorname{Pr}\left(\operatorname{SINR}<2^{\frac{(n+1) u}{W}}-1 \mid n, A\right) & =\operatorname{Pr}\left(\operatorname{SINR}<2^{\frac{(n+1) u}{W}}-1 \mid A\right) \\
& \approx \operatorname{Pr}\left(\operatorname{SINR}<2^{\frac{(n+1) u}{W}}-1\right) .
\end{aligned}
$$

This approximation is reasonable, because Eq. (9) holds equal only when the SINR distribution for any given cell size is the same, and our experimental results show that the SINR distributions in $90 \%$ of cells are similar to each other as shown in Fig. 2. In this figure, we take a one-dimensional homogeneous BS deployment case as an example, and set the BS density 1 . In this scenario, $\operatorname{Pr}(A \in[0.1,2.1])=\int_{0.1}^{2.1} f(A) \mathrm{d} A=0.9$, i.e., the probability of cell size being between 0.1 and 2.1 is $90 \%$. Further for two-dimensional and two-tier cases, the mean cell size $\overline{A_{M}}=\frac{c}{c \rho_{M}+\rho_{m}}$ and $\overline{A_{m}}=\frac{1}{c \rho_{M}+\rho_{m}}$. Through calculations on (6) and (7), we have that both $\operatorname{Pr}\left(A_{M} \in\right.$ $\left.\left[\frac{0.1 c}{c \rho_{M}+\rho_{m}}, \frac{2.1 c}{c \rho_{M}+\rho_{m}}\right]\right)=\int_{0.1 K_{M}}^{2.1 K_{M}} \frac{t^{K_{M}-1}}{\Gamma\left(K_{M}\right)} \exp (-t) \mathrm{d} t$ and $\operatorname{Pr}\left(A_{m} \in\left[\frac{0.1}{c \rho_{M}+\rho_{m}}, \frac{2.1}{c \rho_{M}+\rho_{m}}\right]\right)=\int_{0.1 K_{m}}^{2.1 K_{m}} \frac{t^{K_{m}-1}}{\Gamma\left(K_{m}\right)} \exp (-t) \mathrm{d} t$ are larger than 0.95 . Therefore, most of the cells have similar cell size and thus similar SINR distribution.

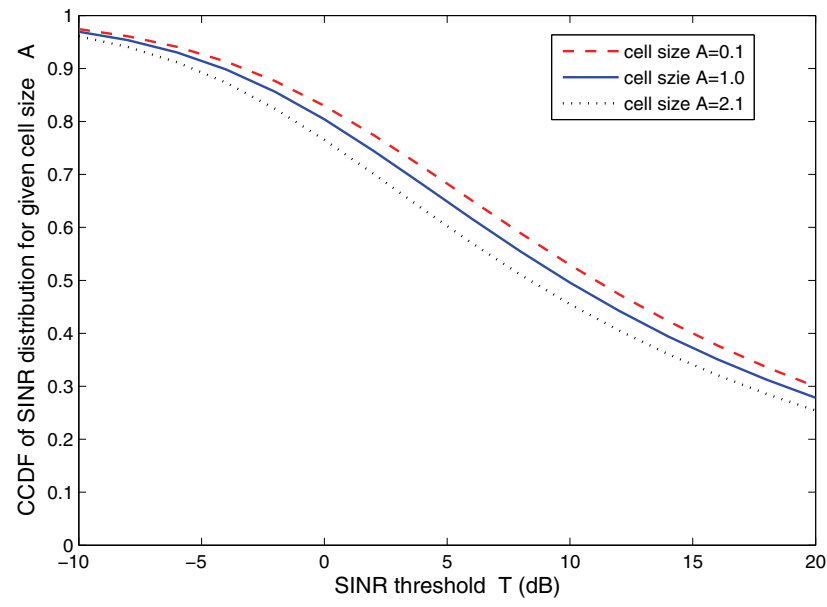

Fig. 2. The complementary cumulative distribution function (CCDF) of the SINR distribution for the given cell size A. About $90 \%$ of cells are with cell size between 0.1 and 2.1 for the one-dimensional homogeneous BS deployment case with BS density 1, and the SINR distributions in these cells are similar to each other.

With this approximation (9), the service outage probability is redefined as

$$
G=\int_{0}^{\infty} \sum_{n=0}^{\infty} \operatorname{Pr}\left(\operatorname{SINR}<2^{\frac{(n+1) u}{W}}-1\right) P_{A}(n) f(A) \mathrm{d} A .
$$

Lemma 1. In the heterogeneous cellular network model with the $\beta$-PSR scheme, the user SINR distribution follows:

$$
\operatorname{Pr}(\operatorname{SINR} \geq T)=\frac{1}{1+\frac{c \rho_{M}+\beta \rho_{m}}{c \rho_{M}+\rho_{m}} T^{2 / \alpha} \int_{T^{-2 / \alpha}}^{\infty} \frac{1}{1+x^{\alpha / 2}} \mathrm{~d} x} .
$$

The detailed proof is shown in Appendix A.

Based on Eqs. (5)-(11), $G_{M}(\beta)$ and $G_{m}(\beta)$ are expressed as (12) and (13), respectively.

\section{B. Main Results}

When the ratio of the user rate requirement $u$ over the whole spectrum bandwidth $W$ is relatively low, the optimal PSR factor $\beta^{*}$ can be accurately approximated by:

$$
\beta^{*} \approx \frac{c \rho_{M}+\rho_{m}+\lambda}{c \rho_{M}+\rho_{m}+c \lambda} .
$$

Remark that $u$ is the minimum service rate requirement per user, not the network throughput. To derive this result, we first need 4 lemmas.

Lemma 2. In the heterogeneous cellular network model with the $\beta$-PSR scheme, $G_{M}(\beta)$ is increasing, while $G_{m}(\beta)$ is decreasing with the PSR factor $\beta$.

The detailed proof of Lemma 2 is shown in Appendix B. Based on Lemma 2 and the fact that $G_{M}(0) \leq G_{m}(0)$ and $G_{M}(1) \geq G_{m}(1)$, we have, 


$$
\begin{aligned}
& G_{M}(\beta)=1-\int_{0}^{\infty} \sum_{n=0}^{\infty} \frac{1}{\left.1+\frac{c \rho_{M}+\beta \rho_{m}}{c \rho_{M}+\rho_{m}}\left(2^{\frac{(n+1) u}{W}}-1\right)^{\frac{2}{\alpha}} \int_{(2}^{\infty} \frac{(n+1) u}{W}-1\right) \frac{-2}{\alpha} \frac{1}{1+x^{\alpha / 2}} \mathrm{~d} x} \frac{(\lambda A)^{n}}{n !} \exp (-\lambda A) A^{K_{M}-1} \frac{\exp \left(-\frac{c \rho_{M}+\rho_{m}}{c} K_{M} A\right)}{\left(\frac{1}{c \rho_{M}+\rho_{m}} \frac{1}{K_{M}}\right)^{K_{M}} \Gamma\left(K_{M}\right)} \mathrm{d} A, \\
& G_{m}(\beta)=1-\int_{0}^{\infty} \sum_{n=0}^{\infty} \frac{1}{\left.1+\frac{c \rho_{M}+\beta \rho_{m}}{c \rho_{M}+\rho_{m}}\left(2^{\frac{(n+1) u}{\beta W}}-1\right)^{\frac{2}{\alpha}} \int_{\left(2{ }_{(2+1) u}^{\infty}\right.}^{\infty}-1\right) \frac{-2}{\alpha} \frac{1}{1+x^{\alpha / 2}} \mathrm{~d} x} \frac{(\lambda A)^{n}}{n !} \exp (-\lambda A) A^{K_{m}-1} \frac{\exp \left(-\left(c \rho_{M}+\rho_{m}\right) K_{m} A\right)}{\left(\frac{1}{c \rho_{M}+\rho_{m}} \frac{1}{K_{m}}\right)^{K_{m}} \Gamma\left(K_{m}\right)} \mathrm{d} A .
\end{aligned}
$$

Lemma 3. In the problem (3), the optimal PSR factor $\beta^{*}$ is achieved if and only if,

$$
G_{M}\left(\beta^{*}\right)=G_{m}\left(\beta^{*}\right) .
$$

Definition 1. Define ' $\lesssim$ ' as a relation between two functions $f(x, y)$ and $g(x, y)$, if the following is satisfied that

$f(x, y) \lesssim_{y} g(x, y) \Leftrightarrow \begin{cases}\lim _{y \rightarrow 0} \frac{f(x, y)}{g(x, y)}=1, & \forall x \in \Omega_{x} ; \\ f(x, y) \leq g(x, y), & \forall\{x, y\} \in \Omega_{x, y},\end{cases}$

where, $\Omega_{x}$ denotes the domain of $x$, and similarly for $\Omega_{x, y}$.

Define ' $\gtrsim$ ' as that $f(x, y) \gtrsim y g(x, y)$ if and only if $g(x, y) \lesssim_{y} f(x, y)$ is satisfied.

Lemma 4. If $f_{1}(x, y) \lesssim_{y} f_{2}(x, y)$ and $f_{2}(x, y) \lesssim_{y} f_{3}(x, y)$, then $f_{1}(x, y) \lesssim_{y} f_{3}(x, y)$.

Lemma 5. In the heterogeneous cellular network model with the $\beta$-PSR scheme, the service outage probabilities $G_{M}(\beta)$ and $G_{m}(\beta)$ have the following properties:

$$
G_{M}(\beta) \lesssim \frac{u}{W} 1-\frac{\frac{1}{\phi} 2^{-\frac{u}{W}} 2^{-\frac{u}{W} \frac{c \lambda}{c \rho_{M}+\rho_{m}}}}{1-\frac{\phi-1}{\phi} 2^{-\frac{u}{W}} 2^{-\frac{u}{W} \frac{c \lambda}{c \rho_{M}+\rho_{m}}}},
$$

and

$$
G_{m}(\beta) \lesssim \frac{u}{W} 1-\frac{\frac{1}{\phi} 2^{-\frac{u}{\beta W}} 2^{-\frac{u}{\beta W} \frac{\lambda}{c \rho_{M}+\rho_{m}}}}{1-\frac{\phi-1}{\phi} 2^{-\frac{u}{\beta W}} 2^{-\frac{u}{\beta W} \frac{\lambda}{c \rho_{M}+\rho_{m}}}},
$$

where, $\phi \triangleq \frac{c \rho_{M}+\beta \rho_{m}}{c \rho_{M}+\rho_{m}} \frac{2}{\alpha-2}$.

The detailed proof of Lemma 5 is shown in Appendix C. With the above lemmas, we have the following theorem to get the asymptotic behavior of the optimal PSR factor.

Theorem 1. In the heterogeneous cellular network model with the $\beta$-PSR scheme, the optimal PSR factor $\beta^{*}$ of the problem (3) has the following property:

$$
\lim _{\frac{u}{w} \rightarrow 0} \beta^{*}=\frac{c \rho_{M}+\rho_{m}+\lambda}{c \rho_{M}+\rho_{m}+c \lambda} .
$$

Proof: According to Lemma 3, the optimal PSR factor $\beta^{*}$ is achieved when $G_{M}\left(\beta^{*}\right)=G_{m}\left(\beta^{*}\right)$. Therefore, with Lemma 5 , as $\frac{u}{W} \rightarrow 0$, the optimal is achieved when

$$
1+\frac{c \lambda}{c \rho_{M}+\rho_{m}}=\frac{1}{\beta^{*}}\left(1+\frac{\lambda}{c \rho_{M}+\rho_{m}}\right),
$$

which leads to Eq. (19). This theorem is proven.

According to Theorem 1, the optimal PSR factor $\beta^{*}$ depends on not only the macro/micro BS density, but also the user intensity. For example, according to Eq. (19), when $\lambda$ is approaching $0, \beta^{*}$ is approaching 1 , but when $\lambda$ is sufficiently large, $\beta^{*}$ approximately equals to $c^{-1}$. The reason for this is that when $\lambda$ is approaching 0 , each user finds himself being the only one user in the cell with the probability 1 . Due to fairness, each user should be allocated with the same bandwidth, and thus $\beta^{*}$ is approaching 1 . On the other hand, when $\lambda$ is approaching infinity, the user number in the macro cells are $c$ times of that in the micro cells with the probability 1. Thus, the micro cell should be allocated with $c^{-1}$ of the network bandwidth.

Theorem 1 also shows that the universal spectrum reuse scheme is optimal for homogeneous networks. Because homogeneous networks are extreme cases of heterogenous networks when the transmit power of macro and micro BSs is the same, i.e., $c=1$. If so, $\beta^{*}$ always equals to 1 , not depending on any system parameters.

With Theorem 1, it is reasonable to have the following observation.

Observation 1. In the heterogeneous cellular network model with the $\beta$-PSR scheme, when $\frac{u}{W}$ is relatively low, the optimal PSR factor $\beta^{*}$ is accurately predicted as

$$
\beta^{*} \approx \frac{c \rho_{M}+\rho_{m}+\lambda}{c \rho_{M}+\rho_{m}+c \lambda} .
$$

With Observation 1, the PSR scheme is easy to realize in a distributed way, because only statistical information is needed. For example, by local information exchange, micro cells can get their cell size, which can be adopted to measure the user density. Then, with system parameters as the macro/micro BS density and their transmit power, the approximate PSR scheme can be performed with near-optimal performance.

\section{Numerical Results}

In this section through numerical results, we will first verify Observation 1, and then answer within what region of $\frac{u}{W}$, the approximation (14) is acceptable.

Fig. 3 shows that with the parameter setting $u=1 \mathrm{Mbps}$, $\mathrm{W}=20 \mathrm{MHz}, \alpha=4, P_{M}=10 P_{m}, \rho_{M}=0.2, \rho_{m}=0.5$, and $\lambda=1$, the optimal PSR factor can be well approximated by $\frac{c \rho_{M}+\rho_{m}+\lambda}{c \rho_{M}+\rho_{m}+c \lambda}$. The blue solid line in this figure shows how the objective function $\max \left\{G_{M}(\beta), G_{m}(\beta)\right\}$ varies with $\beta$ which changes from 0.01 to 1 with the interval 0.01 . Through exhaustive search among these discrete points, the minimal QoS metric is achieved when $\beta=0.50$, which is quite close to our approximate result as $\frac{c \rho_{M}+\rho_{m}+\lambda}{c \rho_{M}+\rho_{m}+c \lambda}=0.4965$. Actually, according to Lemma 2, the target is a unimodal function, which can be solved optimally through the well-known golden section search, and we find in this scenario the optimal PSR factor $\beta^{*}=0.4954$. 


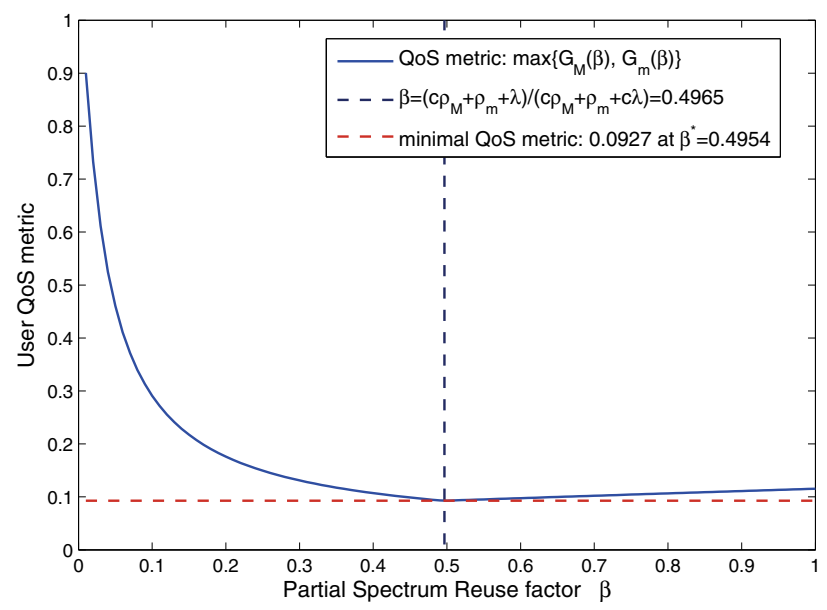

Fig. 3. The optimal PSR factor is accurately predicted by $\frac{c \rho_{M}+\rho_{m}+\lambda}{c \rho_{M}+\rho_{m}+c \lambda}$, with the parameter setting: $\mathrm{u}=1 \mathrm{Mbps}, \mathrm{W}=20 \mathrm{MHz}, \lambda=1, \rho_{M}=0.2, \rho_{m}=0.5$, $\alpha=4, P_{M}=10 P_{m}$.

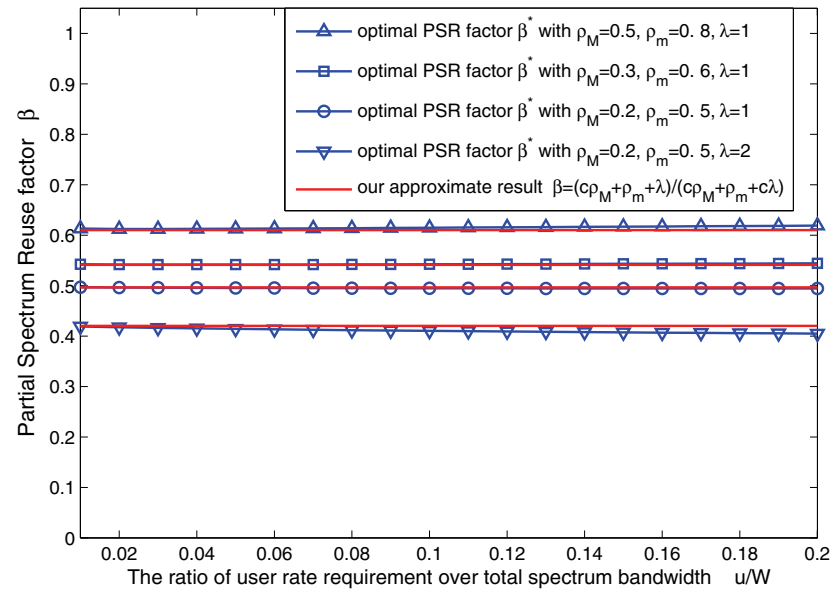

Fig. 4. The optimal PSR factor is accurately predicted by $\frac{c \rho_{M}+\rho_{m}+\lambda}{c \rho_{M}+\rho_{m}+c \lambda}$, with the parameter setting: $\mathrm{u}=0.2: 0.2: 4 \mathrm{Mbps}, \mathrm{W}=20 \mathrm{MHz}, \alpha=4, P_{M}=10 P_{m}$.

Fig. 4 validates the effectiveness of our approximate result further. In this figure, the value of the ratio $\frac{u}{W}$ is changed from 0.01 to 0.2 with the interval 0.01 for different pairs of network density and user density. This figure shows that the error gap between the optimal PSR factor $\beta^{*}$ and the approximate result $\frac{c \rho_{M}+c \rho_{m}+\lambda}{c \rho_{M}+c \rho_{m}+c \lambda}$ is quite small, when the value of $\frac{u}{W}$ goes up to 0.2 (bps/Hz). For example, if the whole spectrum bandwidth $W$ is $20 \mathrm{MHz}$, then the approximation is acceptable when the user rate requirement $u$ is lower than 4 Mbps.

\section{General Case Discussion}

For the general case with different user densities $\left(\lambda_{M}, \lambda_{m}\right)$ and rate requirements $\left(u_{M}, u_{m}\right)$, through some variable substitutions by substituting $\left(\lambda_{M}, u_{M}\right)$ and $\left(\lambda_{m}, u_{m}\right)$ for $(\lambda, u)$ in Lemma 5 respectively, then $G_{M}(\beta)$ and $G_{m}(\beta)$ have the following properties:

$$
G_{M}(\beta) \lesssim_{\frac{1}{W}} 1-\frac{\frac{1}{\phi} 2^{-\frac{u_{M}}{W}} 2^{-\frac{u_{M}}{W} \frac{c \lambda_{M}}{c \rho_{M}+\rho_{m}}}}{1-\frac{\phi-1}{\phi} 2^{-\frac{u_{M}}{W}} 2^{-\frac{u_{M}}{W} \frac{c \lambda_{M}}{c \rho_{M}+\rho_{m}}}},
$$

and

$$
G_{m}(\beta) \lesssim_{\frac{1}{W}} 1-\frac{\frac{1}{\phi} 2^{-\frac{u_{m}}{\beta W}} 2^{-\frac{u_{m}}{\beta W} \frac{\lambda_{m}}{c \rho_{M}+\rho_{m}}}}{1-\frac{\phi-1}{\phi} 2^{-\frac{u_{m}}{\beta W}} 2^{-\frac{u_{m}}{\beta W} \frac{\lambda_{m}}{c \rho_{M}+\rho_{m}}}} .
$$

Therefore, if the objective is still to minimize the value of $\max \left\{G_{M}, G_{m}\right\}$, then the limit of the optimal PSR factor is expressed as

$$
\lim _{W \rightarrow \infty} \beta^{*}=\max \left\{\frac{u_{m}}{u_{M}} \frac{c \rho_{M}+\rho_{m}+\lambda_{m}}{c \rho_{M}+\rho_{m}+c \lambda_{M}}, 1\right\} .
$$

Furthermore, when $\frac{u_{m}}{u_{M}} \frac{c \rho_{M}+\rho_{m}+\lambda_{m}}{c \rho_{M}+\rho_{m}+c \lambda_{M}}>1$ due to high user density or high rate requirement in micro cells, it is better to perform PSR scheme on the macro tier, i.e., each macro cell only occupies a portion $\beta^{\prime}$, while each micro cell occupies the whole wireless spectrum. Through the similar analysis, we have

$$
G_{M}\left(\beta^{\prime}\right) \lesssim_{\frac{1}{W}} 1-\frac{\frac{1}{\phi^{\prime}} 2^{-\frac{u_{M}}{\beta^{\prime} W}} 2^{-\frac{u_{M}}{\beta^{\prime} W} \frac{c \lambda_{M}}{c \rho_{M}+\rho_{m}}}}{1-\frac{\phi^{\prime}-1}{\phi^{\prime}} 2^{-\frac{u_{M}}{\beta^{\prime} W}} 2^{-\frac{u_{M}}{\beta^{\prime} W} \frac{c \lambda_{M}}{c \rho_{M}+\rho_{m}}}},
$$

and

$$
G_{m}\left(\beta^{\prime}\right) \lesssim_{\frac{1}{W}} 1-\frac{\frac{1}{\phi^{\prime}} 2^{-\frac{u_{m}}{W}} 2^{-\frac{u_{m}}{W} \frac{\lambda_{m}}{c \rho_{M}+\rho_{m}}}}{1-\frac{\phi^{\prime}-1}{\phi^{\prime}} 2^{-\frac{u_{m}}{W}} 2^{-\frac{u_{m}}{W}} \frac{\lambda_{m}}{c \rho_{M}+\rho_{m}}},
$$

where $\phi^{\prime}=\frac{\beta^{\prime} c \rho_{M}+\rho_{m}}{c \rho_{M}+\rho_{m}} \frac{2}{\alpha-2}$. Therefore, the limit of the PSR factor $\beta^{\prime}$ is expressed as

$$
\lim _{W \rightarrow \infty} \beta^{*}=\max \left\{\frac{u_{M}}{u_{m}} \frac{c \rho_{M}+\rho_{m}+c \lambda_{M}}{c \rho_{M}+\rho_{m}+\lambda_{m}}, 1\right\} .
$$

To summarize, for the general case, if the system bandwidth is sufficiently large compared to the user rate requirement, then the optimal PSR scheme can be accurately approximated as

$$
\left\{\begin{array}{l}
\frac{W_{m}}{W_{M}}=\frac{u_{m}}{u_{M}} \frac{c \rho_{M}+\rho_{m}+\lambda_{m}}{c \rho_{M}+\rho_{m}+c \lambda_{M}}, \\
\max \left\{W_{M}, W_{m}\right\}=W
\end{array}\right.
$$

where $W_{M}$ and $W_{m}$ denote the bandwidth occupancy for macro and micro cells respectively.

\section{Optimal Network Density Analysis}

Based on the above results, in this section we consider the optimal BS density problem for the heterogeneous cellular network jointly with the PSR schemes, which can be formulated as,

$$
\begin{array}{ll}
\min & \rho_{M}+e \rho_{m} \\
\text { s.t. } & \mathbb{E}_{\left\{N_{M}, A_{M}\right\}}\left\{\operatorname{Pr}\left(\frac{W}{N_{M}} \log _{2}(1+\mathrm{SINR})<u \mid N_{M}, A_{M}\right)\right\}<\eta, \\
& \mathbb{E}_{\left\{N_{m}, A_{m}\right\}}\left\{\operatorname{Pr}\left(\frac{\beta W}{N_{m}} \log _{2}(1+\mathrm{SINR})<u \mid N_{m}, A_{m}\right)\right\}<\eta, \\
& \rho_{0} \leq \rho_{M} \leq \rho_{2}, \\
& \rho_{1} \leq \rho_{m} \leq \rho_{3}, \\
& \beta \in(0,1] .
\end{array}
$$

As Ref. [5] indicates, this problem is motivated by two important practical applications: (1) Capacity extension: how many macro and micro BSs should be deployed based on 
the existing cellular networks. (2) Energy saving: how many macro and micro BSs should be switched off when the traffic load is low, such as at night. For capacity extension, $\left(\rho_{0}, \rho_{1}\right)$ may be the initial existing BS density, while $\left(\rho_{2}, \rho_{3}\right)$ for energy saving. Since coverage is prerequisite, $\rho_{0}$ should be larger than a minimum macro BS density which guarantees the whole network coverage.

\section{A. Problem Analysis}

The original network density problem (29) is quite complex, as even the calculations of $G_{M}(\beta)$ and $G_{m}(\beta)$ are difficult. However, (29) is equivalent to

$$
\begin{array}{cl}
\underset{\left\{\rho_{M}, \rho_{m}, \beta\right\}}{\min } & \rho_{M}+e \rho_{m} \\
\text { s.t. } & \max \left\{G_{M}, G_{m}\right\}<\eta, \\
& \rho_{0} \leq \rho_{M} \leq \rho_{2}, \\
& \rho_{1} \leq \rho_{m} \leq \rho_{3}, \\
& \beta \in(0,1],
\end{array}
$$

which can be further decomposed into two sub-problems as

$$
\begin{array}{cl}
\underset{\left\{\rho_{M}, \rho_{m}\right\}}{\min } & \rho_{M}+e \rho_{m} \\
\text { s.t. } & F^{*}\left(\rho_{M}, \rho_{m}\right)<\eta, \\
& \rho_{0} \leq \rho_{M} \leq \rho_{2}, \\
& \rho_{1} \leq \rho_{m} \leq \rho_{3},
\end{array}
$$

and

$$
\begin{gathered}
F^{*}\left(\rho_{M}, \rho_{m}\right)=\min _{\beta} \max \left\{G_{M}(\beta), G_{m}(\beta)\right\} \\
\text { s.t. } \quad \beta \in(0,1] .
\end{gathered}
$$

Please remark that the decomposed sub-problem (32) is just the optimal PSR scheme formulation (3) in Section III. Therefore, the derived PSR scheme is also optimal in terms of minimizing the energy cost. To make the analysis tractable, we reinforce the sub-problem (31) with Lemma 5 and Theorem 1 as

$$
\begin{aligned}
& \min _{\left\{\rho_{M}, \rho_{m}\right\}} \rho_{M}+e \rho_{m} \\
& \text { s.t. } \frac{\frac{1}{\phi} 2^{-\frac{u}{W}} 2^{-\frac{u}{W} \frac{c \lambda}{c \rho_{M}+\rho_{m}}}}{1-\frac{\phi-1}{\phi} 2^{-\frac{u}{W}} 2^{-\frac{u}{W} \frac{c \lambda}{c \rho_{M}+\rho_{m}}}} \geq 1-\eta \text {, } \\
& \rho_{0} \leq \rho_{M} \leq \rho_{2} \text {, } \\
& \rho_{1} \leq \rho_{m} \leq \rho_{3},
\end{aligned}
$$

where,

$$
\phi=\frac{\left(c \rho_{M}+\rho_{m}\right)^{2}+\left(c^{2} \rho_{M}+\rho_{m}\right) \lambda}{\left(c \rho_{M}+\rho_{m}\right)^{2}+\left(c^{2} \rho_{M}+c \rho_{m}\right) \lambda} \frac{2}{\alpha-2} .
$$

By letting the first constraint hold equal, we have,

$$
\rho_{M}=\frac{c \lambda}{c+\tau} \frac{1}{\frac{W}{u} \log _{2} \frac{\phi^{-1}+(1-\eta)\left(1-\phi^{-1}\right)}{1-\eta}-1} .
$$

Therefore, the reinforced problem can be further equivalent to

$$
\begin{array}{ll}
\min _{\left\{\rho_{M}, \rho_{m}\right\}} & \frac{1+e \tau}{1+\frac{1}{c} \tau} \frac{\lambda}{\frac{W}{u} \log _{2} \frac{\phi^{-1}+(1-\eta)\left(1-\phi^{-1}\right)}{1-\eta}-1} \\
\text { s.t. } & \rho_{0} \leq \frac{c \lambda}{c+\tau} \frac{1}{\frac{W}{u} \log _{2} \frac{\phi^{-1}+(1-\eta)\left(1-\phi^{-1}\right)}{1-\eta}-1} \leq \rho_{2}, \\
& \rho_{1} \leq \frac{c \lambda}{c+\tau} \frac{\tau}{\frac{W}{u} \log _{2} \frac{\phi^{-1}+(1-\eta)\left(1-\phi^{-1}\right)}{1-\eta}-1} \leq \rho_{3} .
\end{array}
$$

If the universal reuse scheme is adopted, i.e., $\phi$ is a fixed value since $\beta=1$, the energy cost function is an increasing function when $e>c^{-1}$, and a decreasing function otherwise. In other words, if $e>c^{-1}$, the optimal is to let $\tau$ as low as possible; otherwise, let $\tau$ as high as possible.

However, if the optimal PSR scheme is adopted, this problem is much more complicated, as $\tau$ and $\phi$ are related with each other. To get the relation between them, by rearranging Eqs. (34) and (35) jointly, we get the following equation,

$\tau=\tau(\phi)=\frac{c\left(1-\frac{\alpha-2}{2} \phi\right) \frac{W}{u} \log _{2} \frac{\phi^{-1}+(1-\eta)\left(1-\phi^{-1}\right)}{1-\eta}}{\left(\frac{\alpha-2}{2} \phi-\frac{1}{c}\right) \frac{W}{u} \log _{2} \frac{\phi^{-1}+(1-\eta)\left(1-\phi^{-1}\right)}{1-\eta}+\frac{1}{c}-1}$.

in which, $\tau$ can be viewed as a function of $\phi$.

Remark that the ratio $\tau$ is positive, therefore the denominator of the expression (37) should also be positive, demonstrating the region in which $\phi$ should be valued.

\section{B. Main Results}

When Eq. (37) is applied to the reinforced problem (36), (36) becomes a one-dimensional optimization problem. In the following, for clarity, we use the symbol $\Omega_{\tau}$ to represent the feasible set of $\tau$, and similarly for $\Omega_{\phi}$. In the reinforced problem (36),

$$
\begin{aligned}
\Omega_{\tau}=\left\{\tau: \rho_{0}\right. & \leq \frac{c \lambda}{c+\tau} \frac{1}{\frac{W}{u} \log _{2} \frac{\phi^{-1}+(1-\eta)\left(1-\phi^{-1}\right)}{1-\eta}-1} \leq \rho_{2} \\
\rho_{1} & \left.\leq \frac{c \lambda}{c+\tau} \frac{\tau}{\frac{W}{u} \log _{2} \frac{\phi^{-1}+(1-\eta)\left(1-\phi^{-1}\right)}{1-\eta}-1} \leq \rho_{3} .\right\},
\end{aligned}
$$

where, $\tau$ and $\phi$ are connected through Eq. (37).

Lemma 6. The function $\tau(\phi)$ is a strictly-decreasing function.

Proof: In the expression (37), the numerator is a strictlydecreasing function. Then we only need to prove that the denominator is an increasing function. $y_{1}=\phi \log _{2}\left(1+\frac{\eta}{1-\eta} \phi^{-1}\right)$ is an increasing function, and $y_{2}=-\log _{2}\left(1+\frac{\eta}{1-\eta} \phi^{-1}\right)$ is also increasing with $\phi$. Therefore, the denominator $\frac{2}{\alpha-2} y_{1}+$ $\frac{1}{c} y_{2}+\frac{1}{c}-1$ is an increasing function, and this lemma has been proven.

Lemma 6 shows $\tau$ and $\phi$ have a one-to-one relationship, and thus $\phi$ can also be viewed as a function of the density ratio $\tau$, as $\phi(\tau)$.

Theorem 2. In the heterogeneous cellular network model with the $\beta$-PSR scheme, on the condition that the ratio of the micro$B S$ energy cost over the macro-BS energy cost is less than $c^{-1}$, 
i.e., $e \leq c^{-1}$, then the optimal network density is achieved when the density ratio $\tau$ gets as high as possible within the feasible set $\Omega_{\tau}$, which is,

$$
\tau^{*}=\max \left\{\Omega_{\tau}\right\} \text {, when } e \leq c^{-1} .
$$

Proof: We only need to prove the objective function is a non-increasing function of $\tau$ on the condition of $e \leq c^{-1}$. First, we have the function $\frac{1+e \tau}{1+\frac{1}{c} \tau}$ is a decreasing function. Then, according to Lemma $6, \phi$ is a strictly-decreasing function of $\tau$. Thus, $\log _{2}\left(1+\frac{\eta}{1-\eta} \phi^{-1}\right)$ is an increasing function with $\tau$, and this theorem has been proven.

According to Theorem 2, when $e \leq c^{-1}$, to let $\tau$ as high as possible, either $\rho_{M}^{*}=\rho_{0}$ or $\rho_{m}^{*}=\rho_{3}$ is satisfied, depending on which determines the feasible region.

Intuitively, the optimal rule has the following structure:

$$
\tau^{*}= \begin{cases}\min \left\{\Omega_{\tau}\right\}, & e>\delta_{2} \\ \max \left\{\Omega_{\tau}\right\}, & e<\delta_{1}\end{cases}
$$

For the universal reuse scheme, $\delta_{1}=\delta_{2}=c^{-1}$. However, when the optimal PSR scheme is adopted, at least we have $\delta_{1}>c^{-1}$, and the gap between them shows how the PSR scheme benefits the micro BSs in terms of larger region in which the micro BSs dominate the macro. Since $\phi(\tau)$, the inverse function of $\tau(\phi)$ (37), is not explicit, it is difficult to get $\delta_{1}$ and $\delta_{2}$ in closed forms, but the following Theorem 3 reveals that in some scenarios, $\delta_{1}$ and $\delta_{2}$ are equal to each other, i.e., $\delta_{1}=\delta_{2}$. For clarity, we use some mathematical symbols to denote some expressions as the following:

$$
B=\frac{W}{u} \log _{2}\left(1+\frac{\eta}{1-\eta} \phi^{-1}\right)-1 ; \quad b=e c-1 .
$$

Theorem 3. In the heterogeneous cellular network model with the $\beta$-PSR scheme, on the condition that,

$$
\frac{1}{B}\left(\frac{\partial B}{\partial \phi}\right)^{2} \leq \frac{\partial^{2} B}{\partial \phi^{2}}, \quad \forall \phi \in\left\{\phi: \frac{\partial f}{\partial \phi}=0, \tau(\phi) \geq 0\right\},
$$

then $f$, the objective function of the reinforced problem (36), i.e., $f=\frac{1+e \tau}{1+c^{-1} \tau} \frac{\lambda}{B}$, is a quasi-concave function of $\phi$.

The detailed proof of Theorem 3 is shown in Appendix D.

Remark that quasi-concave functions have an important property:

$$
f(\phi) \geq \min \left\{f\left(\phi_{\min }\right), f\left(\phi_{\max }\right)\right\}, \quad \forall \phi \in\left[\phi_{\min }, \phi_{\max }\right] .
$$

Therefore to minimize the quasi-concave objective function, the optimal $\phi^{*}$ is either $\phi_{\max }$ or $\phi_{\min }$. With Lemma 6 that $\tau$ is strictly decreasing with $\phi$, we have the following observation.

Observation 2. In the heterogeneous cellular network model with the $\beta$-PSR scheme, on the condition that (42) is satisfied, the optimal density ratio $\tau^{*}$ is either $\max \left\{\Omega_{\tau}\right\}$ or $\min \left\{\Omega_{\tau}\right\}$, i.e.,

$$
\tau^{*}=\left\{\begin{array}{cc}
\min \left\{\Omega_{\tau}\right\}, & e>\delta \\
\max \left\{\Omega_{\tau}\right\}, & e \leq \delta
\end{array}\right.
$$

where, $\delta$ is the threshold of e which satisfies $f\left(\min \left\{\Omega_{\tau}\right\}\right)=$ $f\left(\max \left\{\Omega_{\tau}\right\}\right)$.

Observation 2 shows that when the condition (42) holds, the optimal strategy is to let the density ratio $\tau$ be either as high or as low as possible, depending on whether the cost ratio $e$ exceeds the threshold $\delta$ or not. Specifically, if $e$ is lower than $\delta$, the optimal strategy is to deploy micro BSs or to switch off macro BSs with higher priority. Otherwise, the optimal choice is the opposite. The threshold $\delta$ divides the value space of $e$ into two regions, and within each region, the variation of $e$ has no impact on the optimal BS density and related PSR scheme. Therefore, the PSR scheme and associated energy reduction performance are robust to the variation of $e$ when $e$ varies within a small region.

With Observation 2, the threshold $\delta$ is easy to calculate, greatly depending on the feasible set $\Omega_{\tau}$, as $\delta$ is the value of $e$ to make the equation $f\left(\min \left\{\Omega_{\tau}\right\}\right)=f\left(\max \left\{\Omega_{\tau}\right\}\right)$ hold. Generally, the condition (42) is easy to check, as it only requires to be satisfied at the particular discrete points meeting $\frac{\partial f}{\partial \phi}=0$. Furthermore, the condition (42) is satisfied when the user rate requirement is relatively low. For example, one sufficient condition for (42) is $\frac{W}{u} \frac{\eta}{(1-\eta) \log 2} \leq \frac{W}{u} \frac{2}{c} \log _{2}(1+$ $\left.\frac{c \eta}{1-\eta}\right)+\frac{W}{u} \log _{2}\left(1+\frac{\eta}{1-\eta}\right) \frac{\eta}{1-\eta}-2-\frac{\eta}{1-\eta}$, which holds for all $\eta \in[0.1,1)$ when $W=20 \mathrm{MHz}, u \leq 1 \mathrm{Mbps}$, and $c=3.1623$. Remark that (42) is just one of sufficient conditions to guarantee the quasi-concave property. Therefore, the optimal rule (44) holds for most of current rate requirements.

\section{Numerical Results and Discussions}

In this section, we provide numerical results to justify our reinforced problem analysis, and to evaluate the gap between the optimal results of the original and reinforced problems. We first set up a capacity extension scenario as an example, based on which the comparison between these optimal results is shown in Fig. 5. Finally, with the same scenario, Fig. 6 shows how much energy consumption can be reduced by the PSR scheme, and how much larger dominant region that the optimal PSR scheme brings to the micro BS sites, compared with the scenario without PSR scheme.

We consider a scenario that there have been already deployed with macro BSs with the density $\rho_{0}=0.1$ and micro BSs with the density $\rho_{1}=0.3$, which was planned for the user density $\lambda=1$. However, $\lambda$ has been increased to 2 , requiring capacity extension to satisfy users' QoS requirement. The other parameter settings of the scenario are: the minimum instantaneous service rate requirement $u=1 \mathrm{Mbps}$, the system bandwidth $W=20 \mathrm{MHz}$, the service outage probability threshold $\eta=0.1$, the path loss exponent $\alpha=4$, and the transmit power of macro BSs is $10 \mathrm{~dB}$ higher than that of micro BSs. In the following figures, all the optimal solutions are obtained through the exhaustive search algorithm.

Fig. 5 verifies that the reinforced problem analysis is conservative and effective. From this figure, we can see that the optimal results have the similar trends. All of them have critical points of the cost ratio $e$ approximately equaling to 0.8. In each region below or beyond the critical point, the optimal network energy consumption is linear with $e$, for the optimal macro/micro density solutions are the same. However, the slopes of these two regions are different, e.g., one is much larger than the other. The reason is that one is to let $\tau$ as high as possible, while as low as possible for the other. It is also validated that the performance of the approximate PSR scheme $\beta=\frac{c \rho_{M}+\rho_{m}+\lambda}{c \rho_{M}+\rho_{m}+c \lambda}$ is quite close to that of the optimal PSR scheme. 


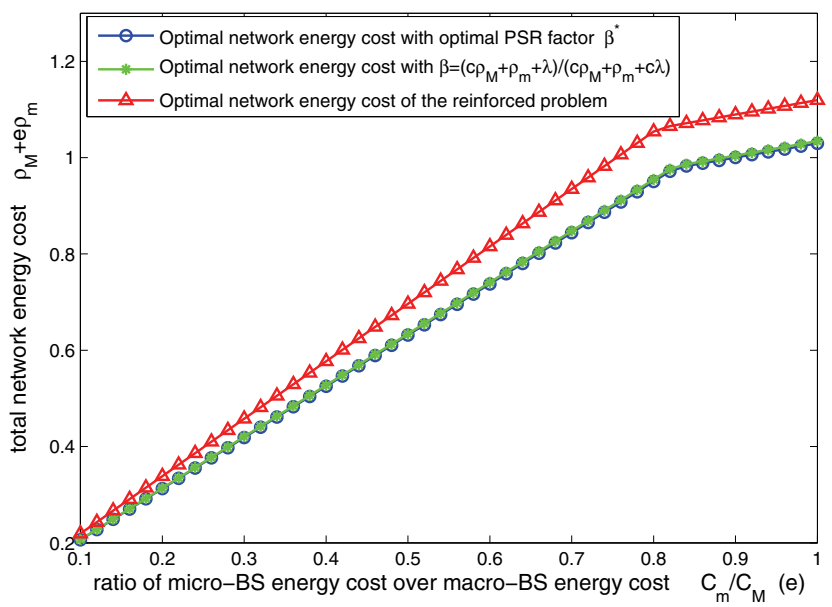

Fig. 5. Optimal network energy consumption of the original density problems (29) with the perfect PSR scheme, the approximate PSR scheme $\beta=\frac{c \rho_{M}+\rho_{m}+\lambda}{c \rho_{M}+\rho_{m}+c \lambda}$, and the reinforced problem (33). A capacity extension example with the parameter setting: $u=1 \mathrm{Mbps}, W=20 \mathrm{MHz}, \eta=0.1$, $P_{M}=10 P_{m}, \alpha=4, \lambda=2, \rho_{0}=0.1, \rho_{1}=0.3, \rho_{2}=\rho_{3}=\infty$. It shows that the approximate PSR scheme achieves the near-optimal performance.

In Fig. 6, we provide a comparison between the optimal network energy consumptions of the optimal PSR scheme and the non-PSR scheme. From this figure, we can see that for both schemes, there exists a threshold of the cost ratio $e$ to determine whether micro BS sites are preferable or not. Specifically, there is a critical point in both lines, and the $\mathrm{x}$-coordinate of the critical point denotes the threshold. The threshold $\delta$ divides the value space of $e$ into two regions $(0, \delta]$ and $(\delta, \infty)$. In each region, the optimal BS density $\left(\rho_{M}^{*}, \rho_{m}^{*}\right)$ is the same according to Observation 2, and thus the minimum energy cost $\rho_{M}^{*}+e \rho_{m}^{*}$ is a linear function of $e$ with the slope $\rho_{m}^{*}$ and the y-intercept $\rho_{M}^{*}$. Please note that the slopes between these two regions are different. The reason for this difference is that in the first region, the optimal choice is to let the density ratio $\tau$ be as high as possible, i.e., to only add more micro BSs (because $\rho_{3}$ is infinite) and keep the macro BS density constant, while in the second region, the optimal choice is the opposite. Since more micro BSs are required, the slope in the first region is larger than that in the second region, as shown in this figure.

Theorem 2 proves that the threshold of the PSR scheme is larger than that of the non-PSR scheme. The gap between these two thresholds shows that the micro BS sites dominate the macro BSs in a much larger region, as the optimal PSR scheme can greatly reduce the required micro BS density. Fig. 6 also reveals that it is crucial to adopt PSR schemes when $e \approx c^{-1}$, for it can save the most network energy cost, up to $50 \%$.

The energy cost reduction gain of the PSR scheme, greatly depends on specific parameter settings such as transmit power $\left(P_{M}, P_{m}\right)$, user density $\lambda$ and others. Remark that the nonPSR scheme, i.e., the universal spectrum reuse scheme, is a specific $\beta$-PSR scheme in which the PSR factor $\beta$ always equals to 1 . Since the service outage performance is decreasing with $\beta$ in the region $\left[\beta^{*}, 1\right]$, it is reasonable to conjecture that smaller gap between $\beta^{*}$ and 1 leads to smaller energy reduc-

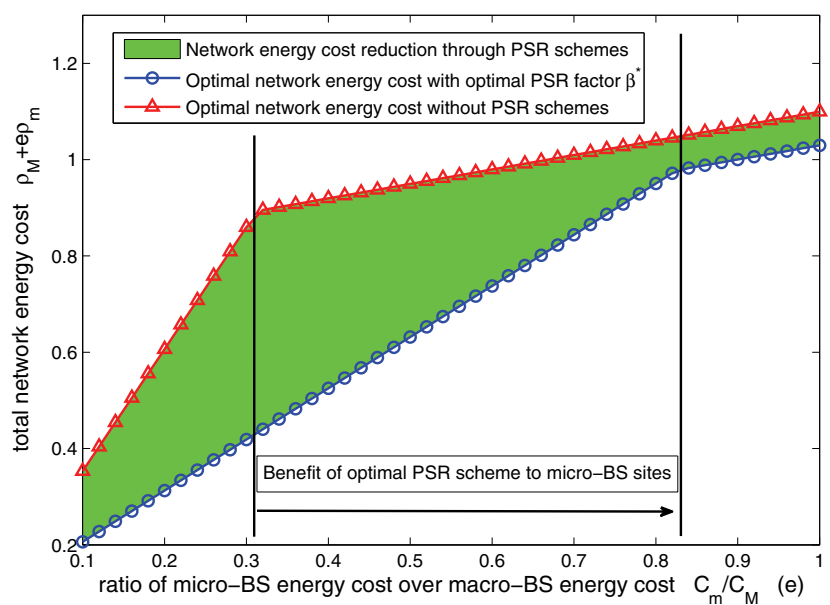

Fig. 6. Energy saving gain of the PSR scheme and the benefit of PSR to micro-BS sites. A capacity extension example with the parameter setting: $u=1 \mathrm{Mbps}, W=20 \mathrm{MHz}, \eta=0.1, P_{M}=10 P_{m}, \alpha=4, \lambda=2$, $\rho_{0}=0.1, \rho_{1}=0.3, \rho_{2}=\rho_{3}=\infty$. The optimal PSR scheme can greatly reduce the required density of micro BSs, thus benefitting the micro BS sites in terms of a much larger region to be preferable.

tion gain. For example, consider an extreme case with the same transmit power of macro and micro BSs, i.e., $c=\left(\frac{P_{M}}{P_{m}}\right)^{2 / \alpha}=1$. In such case, the gap between $\beta^{*}$ and 1 is 0 , and the energy reduction gain is also 0 . The expression $\beta^{*} \approx \frac{c \rho_{M}+\rho_{m}+\lambda}{c \rho_{M}+\rho_{m}+c \lambda}$ reveals that if other parameters keep constant, larger user density $\lambda$ and larger system parameter $c$ lead to smaller $\beta^{*}$, and thus larger energy reduction gain.

\section{CONCLUSION}

In this paper, we derive the optimal PSR scheme, and analyze the BS density problem jointly with PSR schemes in heterogeneous cellular networks. Based on the asymptotic analysis of service outage probability, when the ratio of the user rate requirement over the whole spectrum bandwidth is approaching zero, we derive a closed-form limit of the optimal PSR factor, which is a function of traffic intensity, the macro/micro BS density and their transmit power. Our numerical results show that at least when the ratio of user rate requirement over the system bandwidth is lower than 0.2 $\mathrm{bps} / \mathrm{Hz}$, this approximation is acceptable. Based on the above results, it is derived that generally the network energy cost is quasi-concave. Therefore, there exists a threshold of the micro BS energy cost to determine which type of BSs is preferable. When the micro-BS energy cost is below this threshold, the optimal strategy is to deploy more micro BSs or to switch off more macro BSs; otherwise, the optimal choice is the opposite. This threshold is definitely larger than that without PSR scheme. Our numerical results also show that the PSR scheme can save up to $50 \%$ of network energy consumption when the transmit power of micro BSs is $10 \mathrm{~dB}$ lower than that of macro BSs, compared with no PSR schemes.

\section{APPENDIX A \\ PROOF OF LEMMA 1}

Proof: As proven in Ref. [25], in the Rayleigh fading, noise-free and homogeneous PPP BS deployment scenario, the 
coverage probability that the SINR of a random user is larger than a threshold $T$ with spectrum reuse factor $\delta(\delta \in(0,1])$ is expressed as

$$
\operatorname{Pr}(\operatorname{SINR} \geq T)=\frac{1}{1+\delta T^{2 / \alpha} \int_{T^{-2 / \alpha}}^{\infty} \frac{1}{1+x^{\alpha / 2}} \mathrm{~d} x},
$$

which is independent with the BS density, as the noise is ignored.

Note that the formula (45) is based on the homogeneous PPP model. In the heterogeneous scenario, for any given user, a macro BS with distance $d_{M}$ is equivalent to a micro BS with shorter distance $d_{m}=\left(\frac{P_{m}}{P_{M}}\right)^{1 / \alpha} d_{M}$ just on the received signal strength level. Therefore, for any given user, the macro BSs with the density $\rho_{M}$ can be equivalent to micro BSs deployed according to a PPP with the density $c \rho_{M}$ if only signal strength is considered. Since the superposition of two and more independent PPPs is still a PPP [24], the heterogeneous cellular network is equivalent to the homogeneous network with density $c \rho_{M}+\rho_{m}$. In the equivalent homogeneous network, the interfering BSs occupy the same spectrum with the probability $\frac{c \rho_{M}+\beta \rho_{m}}{c \rho_{M}+\rho_{m}}$. By letting $\delta=\frac{c \rho_{M}+\beta \rho_{m}}{c \rho_{M}+\rho_{m}}$ in the formula (45), we prove this lemma.

\section{APPENDIX B}

\section{PROOF OF LEMMA 2}

Proof: $G_{M}(\beta)$ is an increasing function, because increasing $\beta$, will bring the more inter-cell interference from the micro BSs, as Eq. (11) shows. Specifically, the function $y_{0}=\beta\left(2^{a}-1\right)^{2 / \alpha} \int_{\left(2^{a}-1\right)^{-2 / \alpha}}^{+\infty} \frac{1}{1+x^{\alpha / 2}} \mathrm{~d} x$ is increasing with $\beta$ for all $a \in(0,+\infty)$. By letting $a=(n+1) \frac{u}{W}$, we can prove the monotonicity of $G_{M}(\beta)$.

Similarly to prove that $G_{m}(\beta)$ is decreasing with $\beta$, we only need to prove that for all $a \in(0,+\infty)$, the function $y=\beta T^{2 / \alpha} \int_{T^{-2 / \alpha}}^{+\infty} \frac{1}{1+x^{\alpha / 2}} \mathrm{~d} x$ is decreasing with $\beta$, in which $T=2^{\frac{a}{\beta}}-1$.

Denote $T^{2 / \alpha}$ by $t$, and then $y$ can be rewritten as

$$
y=\frac{a}{\log _{2}\left(1+t^{\frac{\alpha}{2}}\right)} t \int_{t^{-1}}^{+\infty} \frac{1}{1+x^{\alpha / 2}} \mathrm{~d} x .
$$

Because $\frac{\partial t}{\partial \beta} \leq 0$ and the value of $a$ has no impact on the monotonicity, we only need to prove that the function $y_{1}=\frac{1}{a \log (2)} y=\frac{1}{\log \left(1+t^{\frac{\alpha}{2}}\right)} t \int_{t^{-1}}^{+\infty} \frac{1}{1+x^{\alpha / 2}} \mathrm{~d} x$ is increasing with $t$, and we have (47).

To prove $\frac{\partial y_{1}}{\partial t} \geq 0$ is equivalent to proving that the function

$$
\begin{aligned}
y_{2} & =\log \left(1+t^{\frac{\alpha}{2}}\right) \frac{\partial y_{1}}{\partial t} \\
& =\left(1-\frac{\frac{\alpha}{2}}{\log \left(1+t^{\frac{\alpha}{2}}\right)} \frac{t^{\frac{\alpha}{2}}}{1+t^{\frac{\alpha}{2}}}\right) \int_{t^{-1}}^{+\infty} \frac{1}{1+x^{\alpha / 2}} \mathrm{~d} x+\frac{1}{t} \frac{t^{\frac{\alpha}{2}}}{1+t^{\frac{\alpha}{2}}}
\end{aligned}
$$

is greater than 0 .

According to (48), if $\left(1-\frac{\frac{\alpha}{2}}{\log \left(1+t^{\frac{\alpha}{2}}\right)} \frac{t^{\frac{\alpha}{2}}}{1+t^{\frac{\alpha}{2}}}\right) \geq 0$, then $y_{2} \geq$ 0 , and this lemma holds. Therefore, we only need to prove that even on the condition $\left(1-\frac{\frac{\alpha}{2}}{\log \left(1+t^{\frac{\alpha}{2}}\right)} \frac{t^{\frac{\alpha}{2}}}{1+t^{\frac{\alpha}{2}}}\right)<0, y_{2}$ is still greater than 0 .

Remark that

$$
\int_{t^{-1}}^{+\infty} \frac{1}{1+x^{\alpha / 2}} \mathrm{~d} x \leq \int_{t^{-1}}^{+\infty} x^{-\alpha / 2} \mathrm{~d} x=\frac{2}{\alpha-2} t^{\frac{\alpha}{2}-1} .
$$

Therefore, on condition $\left(1-\frac{\frac{\alpha}{2}}{\log \left(1+t^{\frac{\alpha}{2}}\right)} \frac{t^{\frac{\alpha}{2}}}{1+t^{\frac{\alpha}{2}}}\right)<0$,

$$
y_{2} \geq y_{3}=\left(1-\frac{\frac{\alpha}{2}}{\log \left(1+t^{\frac{\alpha}{2}}\right)} \frac{t^{\frac{\alpha}{2}}}{1+t^{\frac{\alpha}{2}}}\right) \frac{2}{\alpha-2} t^{\frac{\alpha}{2}-1}+\frac{1}{t} \frac{t^{\frac{\alpha}{2}}}{1+t^{\frac{\alpha}{2}}} .
$$

With (50), to prove $y_{2} \geq 0$ we only need to prove $y_{3} \geq 0$, or equivalently to prove $y_{4}=t \frac{1+t^{\frac{\alpha}{2}}}{t^{\frac{\alpha}{2}}} \log \left(1+t^{\frac{\alpha}{2}}\right) y_{3} \geq 0$.

By letting $s=t^{\frac{\alpha}{2}}$, the function $y_{4}$ can be rewritten as

$$
y_{4}=\frac{2}{\alpha-2}(1+s) \log (1+s)+\log (1+s)-\frac{\alpha}{\alpha-2} s .
$$

Note that $\lim _{s \rightarrow 0} y_{4}=0$, and thus if $y_{4}$ is increasing with $s$, then $y_{4} \geq 0$ holds for all $s \in(0,+\infty)$, and this lemma is proven. Due to $\alpha \in(2,4]$,

$$
\begin{aligned}
\frac{\partial y_{4}}{\partial s} & =\frac{2}{\alpha-2} \log (1+s)-\frac{s}{1+s} \\
& \geq \log (1+s)-\frac{s}{1+s} \\
& \geq 0 .
\end{aligned}
$$

Therefore, Lemma 2 is proven.

\section{APPENDIX C}

\section{PROOF OF LEMMA 5}

Proof: As $\frac{u}{W} \rightarrow 0, T=2^{(n+1) \frac{u}{W}}-1 \rightarrow 0$, and $T^{-2 / \alpha} \rightarrow$ $\infty$ for any given $n$. Therefore, we have,

$$
\begin{aligned}
\operatorname{Pr}(\operatorname{SINR}<T) & =1-\frac{1}{1+\frac{c \rho_{M}+\beta \rho_{m}}{c \rho_{M}+\rho_{m}} T^{2 / \alpha} \int_{T^{-2 / \alpha}}^{\infty} \frac{1}{1+x^{\alpha / 2}} \mathrm{~d} x} \\
& \lesssim_{W} 1-\frac{1}{1+\frac{c \rho_{M}+\beta \rho_{m}}{c \rho_{M}+\rho_{m}} T^{2 / \alpha} \int_{T^{-2 / \alpha}}^{\infty} x^{-\alpha / 2} \mathrm{~d} x} \\
& =1-\frac{1}{1+\frac{c \rho_{M}+\beta \rho_{m}}{c \rho_{M}+\rho_{m}} \frac{2}{\alpha-2} T}=1-\frac{1}{1+\phi T} \\
& =1-\frac{1}{\phi} 2^{-(n+1) \frac{u}{W}} \frac{1}{1+\frac{1-\phi}{\phi} 2^{-(n+1) \frac{u}{W}}} \\
& =1-\frac{1}{\phi} 2^{-(n+1) \frac{u}{W}} \sum_{m=0}^{\infty}\left\{\frac{\phi-1}{\phi} 2^{-(n+1) \frac{u}{W}}\right\}^{m} .
\end{aligned}
$$

Eqs. (12) and (53) jointly lead to the following,

$$
\begin{aligned}
G_{M}(\beta) \lesssim_{\frac{u}{W}} & -\frac{1}{\phi} 2^{-\frac{u}{W}} \sum_{m=0}^{\infty}\left(\frac{\phi-1}{\phi} 2^{-\frac{u}{W}}\right)^{m} \\
& \left\{\frac{\frac{c \rho_{M}+\rho_{m}}{c} K_{M}}{\left(1-2^{-(m+1) \frac{u}{W}}\right) \lambda+\frac{c \rho_{M}+\rho_{m}}{c} K_{M}}\right\}^{K_{M}} .
\end{aligned}
$$

Further, due to the well-known exponential limit,

$$
\begin{aligned}
& \left\{\frac{\frac{c \rho_{M}+\rho_{m}}{c} K_{M}}{\left(1-2^{-(m+1) \frac{u}{W}}\right) \lambda+\frac{c \rho_{M}+\rho_{m}}{c} K_{M}}\right\}^{K_{M}} \\
& =\left\{1+\frac{1}{\frac{1}{\left(1-2^{-(m+1)} \frac{u}{W}\right) \lambda} \frac{c \rho_{M}+\rho_{m}}{c} K_{M}}\right\}^{-K_{M}} \\
& \gtrsim_{W} \exp \left\{-\left(1-2^{-(m+1) \frac{u}{W}}\right) \frac{c \lambda}{c \rho_{M}+\rho_{m}}\right\} .
\end{aligned}
$$

Because the well-known exponential limit reveals that $\left(1+\frac{1}{K_{M} x}\right)^{K_{M} x} \lesssim_{\left(\frac{1}{x}\right)} \exp (1)$, which leads to $\left(1+\frac{1}{K_{M} x}\right)^{-K_{M}}=\left(1+\frac{1}{K_{M} x}\right)^{-K_{M} x\left(\frac{1}{x}\right)} \gtrsim_{\left(\frac{1}{x}\right)} \exp \left(-\frac{1}{x}\right)$. 


$$
\begin{aligned}
\frac{\partial y_{1}}{\partial t}= & \frac{-1}{\log ^{2}\left(1+t^{\frac{\alpha}{2}}\right)} \frac{1}{1+t^{\frac{\alpha}{2}}} \frac{\alpha}{2} t^{\frac{\alpha}{2}} \int_{t^{-1}}^{+\infty} \frac{1}{1+x^{\alpha / 2}} \mathrm{~d} x \\
& +\frac{1}{\log \left(1+t^{\frac{\alpha}{2}}\right)} \int_{t^{-1}}^{+\infty} \frac{1}{1+x^{\alpha / 2}} \mathrm{~d} x+\frac{1}{\log \left(1+t^{\frac{\alpha}{2}}\right)} \frac{1}{1+t^{-\frac{\alpha}{2}}} \frac{1}{t}
\end{aligned}
$$

By letting $x=\frac{1}{\left(1-2^{-(m+1)} \frac{u}{W}\right)} \frac{c \rho_{M}+\rho_{m}}{c \lambda}$, in which $\frac{u}{W} \rightarrow 0 \Rightarrow \frac{1}{x} \rightarrow 0$, we prove $(55)$.

By adopting (55) into (54), we have (56).

Therefore, (17) is proven, and similarly for (18). This lemma is proven.

\section{APPENDIX D \\ PROOF OF THEOREM 3}

Proof: With Theorem 2, we have that when $e \leq c^{-1}$, the objective function is increasing with $\phi$, and thus quasiconcave. In the rest, we only focus on the scenarios in which $e>c^{-1}$, i.e., $b>0$.

With the symbols defined in (41), Eq. (37) can be rewritten as

$$
\begin{aligned}
\tau & =\frac{c\left(1-\frac{\alpha-2}{2} \phi\right) \frac{W}{u} \log _{2} \frac{\phi^{-1}+(1-\eta)\left(1-\phi^{-1}\right)}{1-\eta}}{\left(\frac{\alpha-2}{2} \phi-\frac{1}{c}\right) \frac{W}{u} \log _{2} \frac{\phi^{-1}+(1-\eta)\left(1-\phi^{-1}\right)}{1-\eta}+\frac{1}{c}-1}, \\
& =\frac{c\left(1-\frac{\alpha-2}{2} \phi\right)(B+1)}{\left(\frac{\alpha-2}{2} \phi-\frac{1}{c}\right)(B+1)+\frac{1}{c}-1},
\end{aligned}
$$

which, when applied into the objective function $f$, leads to

$$
\begin{aligned}
f & =\frac{1+e \tau}{1+c^{-1} \tau} \frac{\lambda}{B} \\
& =\frac{1+e \frac{c\left(1-\frac{\alpha-2}{2} \phi\right)(B+1)}{\left(\frac{\alpha-2}{2} \phi-\frac{1}{c}\right)(B+1)+\frac{1}{c}-1}}{1+\frac{\left(1-\frac{\alpha-2}{2} \phi\right)(B+1)}{\left(\frac{\alpha-2}{2} \phi-\frac{1}{c}\right)(B+1)+\frac{1}{c}-1}} \frac{\lambda}{B} \\
& =\frac{\left(\frac{\alpha-2}{2} \phi-\frac{1}{c}\right)(B+1)+\frac{1}{c}-1+(b+1)\left(1-\frac{\alpha-2}{2} \phi\right)(B+1)}{\left(\frac{\alpha-2}{2} \phi-\frac{1}{c}\right)(B+1)+\frac{1}{c}-1+\left(1-\frac{\alpha-2}{2} \phi\right)(B+1)} \frac{\lambda}{B} \\
& =\frac{\left(1-\frac{1}{c}\right) B+b\left(1-\frac{\alpha-2}{2} \phi\right)(B+1)}{\left(1-\frac{1}{c}\right) B} \frac{\lambda}{B} \\
& =\left[\frac{1}{B}+\frac{b\left(1-\frac{\alpha-2}{2} \phi\right)}{\left(1-\frac{1}{c}\right) B}+\frac{b\left(1-\frac{\alpha-2}{2} \phi\right)}{\left(1-\frac{1}{c}\right) B^{2}}\right] \lambda .
\end{aligned}
$$

For clarity, in the following we ignore $\lambda$, because as long as $\lambda>0$, the variety of $\lambda$ has no impact on the quasi-concavity of the objective function. Therefore without loss of generality, we focus on $f=\frac{1}{B}+\frac{b\left(1-\frac{\alpha-2}{2} \phi\right)}{\left(1-\frac{1}{c}\right) B}+\frac{b\left(1-\frac{\alpha-2}{2} \phi\right)}{\left(1-\frac{1}{c}\right) B^{2}}$, and further we have that

$$
\begin{aligned}
f^{\prime}= & -\left[\frac{1}{B^{2}}+\frac{b\left(1-\frac{\alpha-2}{2} \phi\right)}{\left(1-\frac{1}{c}\right) B^{2}}+\frac{2 b\left(1-\frac{\alpha-2}{2} \phi\right)}{\left(1-\frac{1}{c}\right) B^{3}}\right] \frac{\partial B}{\partial \phi} \\
& -\frac{b \frac{\alpha-2}{2}}{\left(1-\frac{1}{c}\right) B}-\frac{b \frac{\alpha-2}{2}}{\left(1-\frac{1}{c}\right) B^{2}},
\end{aligned}
$$

and,

$$
\begin{aligned}
f^{\prime \prime}= & {\left[\frac{2}{B^{3}}+\frac{2 b\left(1-\frac{\alpha-2}{2} \phi\right)}{\left(1-\frac{1}{c}\right) B^{3}}+\frac{6 b\left(1-\frac{\alpha-2}{2} \phi\right)}{\left(1-\frac{1}{c}\right) B^{4}}\right]\left(\frac{\partial B}{\partial \phi}\right)^{2} } \\
& +\left[\frac{2 b \frac{\alpha-2}{2}}{\left(1-\frac{1}{c}\right) B^{2}}+\frac{4 b \frac{\alpha-2}{2}}{\left(1-\frac{1}{c}\right) B^{3}}\right] \frac{\partial B}{\partial \phi} \\
& +\left[-\frac{1}{B^{2}}-\frac{b\left(1-\frac{\alpha-2}{2} \phi\right)}{\left(1-\frac{1}{c}\right) B^{2}}-\frac{2 b\left(1-\frac{\alpha-2}{2} \phi\right)}{\left(1-\frac{1}{c}\right) B^{3}}\right] \frac{\partial^{2} B}{\partial \phi^{2}} .
\end{aligned}
$$

At each particular point $\phi$ that $\frac{\partial f}{\partial \phi}=0$, by applying the condition (42) into (60), we can further get,

$$
\begin{aligned}
f^{\prime \prime} \leq & {\left[\frac{1}{B^{3}}+\frac{b\left(1-\frac{\alpha-2}{2} \phi\right)}{\left(1-\frac{1}{c}\right) B^{3}}+\frac{4 b\left(1-\frac{\alpha-2}{2} \phi\right)}{\left(1-\frac{1}{c}\right) B^{4}}\right]\left(\frac{\partial B}{\partial \phi}\right)^{2} } \\
& +\left[\frac{2 b \frac{\alpha-2}{2}}{\left(1-\frac{1}{c}\right) B^{2}}+\frac{4 b \frac{\alpha-2}{2}}{\left(1-\frac{1}{c}\right) B^{3}}\right] \frac{\partial B}{\partial \phi} .
\end{aligned}
$$

According to the second-order condition of quasi-convex functions that "as a (partial) converse, if $f$ satisfies $f^{\prime}=0 \Rightarrow$ $f^{\prime \prime}>0$, for all $x \in \operatorname{dom} f$, then $f$ is quasi-convex", (Page 101 in [30]), we only need to prove that $f^{\prime}=0$ always leads to $f^{\prime \prime}<0$.

By letting $f^{\prime}=0$, we have,

$$
\frac{\partial B}{\partial \phi}=\frac{-\frac{b \frac{\alpha-2}{2}}{\left(1-\frac{1}{c}\right)}-\frac{b \frac{\alpha-2}{2}}{\left(1-\frac{1}{c}\right) B}}{\frac{1}{B}+\frac{b\left(1-\frac{\alpha-2}{2} \phi\right)}{\left(1-\frac{1}{c}\right) B}+\frac{2 b\left(1-\frac{\alpha-2}{2} \phi\right)}{\left(1-\frac{1}{c}\right) B^{2}}}<0,
$$

which, when applied into the expression (61), leads to (63).

Therefore, this theorem has been proven.

\section{REFERENCES}

[1] Available: http://it.icxo.com/htmlnews/2010/05/14/1383534.htm

[2] A. Damnjanovic, et al., "A survey on 3GPP heterogeneous networks" (invited paper), IEEE Wireless Commun., vol. 18, no. 3, pp. 10-21, June 2011.

[3] Qualcomm Inc., "LTE advanced: heterogeneous networks," white paper, Jan. 2011. Available: http://www.qualcomm.com/documents/ lte-advanced-heterogeneous-networks- 0 .

[4] R. Madan, J. Borran, A. Sampath, N. Bhushan, A. Khandekar, and T. Ji, "Cell association and interference coordination in heterogeneous LTEA cellular networks," IEEE J. Sel. Areas Commun., vol. 28, no. 9, pp. 1479-1489, Dec. 2010.

[5] D. Cao, S. Zhou, and Z. Niu, "Optimal base station density for energyefficient heterogeneous cellular networks," Proc. 2012 IEEE ICC.

[6] J. O'Carroll, H. Claussen, and L. Doyle, "Partial GSM spectrum reuse for femtocells," Proc. 2009 IEEE PIMRC.

[7] V. Chandrasekhar, J. G. Andrews, and A. Gatherer, "Femtocell networks: a survey," IEEE Commun. Mag., vol. 46, no. 9, pp. 59-67, Sept. 2008.

[8] S. Ali and V. Leung, "Dynamic frequency allocation in frational frequncy reused OFDMA networks," IEEE Trans. Wireless Commun., vol. 8, no. 8, Aug. 2009.

[9] R. Chang, Z. Tao, J. Zhang, and C. Kuo, "A graph approach to dynamic fractional frequency reuse (FFR) in multi-cell OFDMA networks," Proc. 2009 IEEE ICC.

[10] T. Novlan, R. Ganti, A. Ghosh, and J. Andrews, "Analytical evaluation of fractional frequency reuse for OFDMA cellular networks," IEEE Trans. Wireless Commun., vol. 10, no. 12, pp. 4294-4305, 2011.

[11] M. A. Marsan, L. Chiaraviglio, D. Ciullo, and M. Meo, "Optimal energy saving in cellular access networks," 2009 IEEE ICC.

[12] S. Zhou, J. Gong, Z. Yang, Z. Niu, and P. Yang, "Green mobile access network with dynamic base station energy saving," in Proc. 2009 ACM MobiCom (poster).

[13] Z. Niu, Y. Wu, J. Gong, and Z. Yang, "Cell zooming for cost-efficient green cellular networks," IEEE Commun. Mag., vol. 48, no. 11, pp. 74-79, Nov. 2010.

[14] F. Richter, A. J. Febske, and G. P. Fettweis, "Energy efficiency aspects of base station deployment strategies in cellular networks," in Proc. 2009 IEEE VTC - Fall.

[15] B. Badic, T. O'Farrel, P. Loskot, and J. He, "Energy efficiency radio access architectures for green radio: large versus small cell size deployment," in Proc. 2009 IEEE VTC - Fall. 


$$
\begin{aligned}
& G_{M}(\beta) \\
& \lesssim_{\frac{u}{W}} 1-\frac{1}{\phi} 2^{\frac{-u}{W}} \sum_{m=0}^{\infty}\left(\frac{\phi-1}{\phi}\right)^{m} 2^{\frac{-m u}{W}} \exp \left\{-\left(1-2^{\frac{-(m+1) u}{W}}\right) \frac{c \lambda}{c \rho_{M}+\rho_{m}}\right\} \\
& \lesssim_{\frac{u}{W}} 1-\frac{1}{\phi} 2^{\frac{-u}{W}} \sum_{m=0}^{\infty}\left(\frac{\phi-1}{\phi}\right)^{m} 2^{\frac{-m u}{W}} \exp \left\{\frac{-(m+1) u}{W} \log 2 \frac{c \lambda}{c \rho_{M}+\rho_{m}}\right\} \\
& =1-\frac{1}{\phi} 2^{-\frac{u}{W}} 2^{-\frac{u}{W} \frac{c \lambda}{c \rho_{M}+\rho_{m}}} \sum_{m=0}^{\infty}\left\{\frac{\phi-1}{\phi} 2^{-\frac{u}{W}} 2^{-\frac{u}{W} \frac{c \lambda}{c \rho_{M}+\rho_{m}}}\right\}^{m} \\
& =1-\frac{\frac{1}{\phi} 2^{-\frac{u}{W}} 2^{-\frac{u}{W} \frac{c \lambda}{c \rho_{M}+\rho_{m}}}}{1-\frac{\phi-1}{\phi} 2^{-\frac{u}{W}} 2^{-\frac{u}{W} \frac{c \lambda}{c \rho_{M}+\rho_{m}}}} .
\end{aligned}
$$

$$
\begin{aligned}
f^{\prime \prime} \leq & \frac{1}{B^{2}} \frac{\partial B}{\partial \phi}\left\{\frac{\frac{1}{B}+\frac{b\left(1-\frac{\alpha-2}{2} \phi\right)}{\left(1-\frac{1}{c}\right) B}+\frac{4 b\left(1-\frac{\alpha-2}{2} \phi\right)}{\left(1-\frac{1}{c}\right) B^{2}}}{\frac{1}{B}+\frac{b\left(1-\frac{\alpha-2}{2} \phi\right)}{\left(1-\frac{1}{c}\right) B}+\frac{2 b\left(1-\frac{\alpha-2}{2} \phi\right)}{\left(1-\frac{1}{c}\right) B^{2}}}\right. \\
& \left.\left(-\frac{b \frac{\alpha-2}{2}}{\left(1-\frac{1}{c}\right)}-\frac{b \frac{\alpha-2}{2}}{\left(1-\frac{1}{c}\right) B}\right)+\frac{2 b \frac{\alpha-2}{2}}{\left(1-\frac{1}{c}\right)}+\frac{4 b \frac{\alpha-2}{2}}{\left(1-\frac{1}{c}\right) B}\right\} \\
\leq & \frac{1}{B^{2}} \frac{\partial B}{\partial \phi}\left\{2\left(-\frac{b \frac{\alpha-2}{2}}{\left(1-\frac{1}{c}\right)}-\frac{b \frac{\alpha-2}{2}}{\left(1-\frac{1}{c}\right) B}\right)+\frac{2 b \frac{\alpha-2}{2}}{\left(1-\frac{1}{c}\right)}+\frac{4 b \frac{\alpha-2}{2}}{\left(1-\frac{1}{c}\right) B}\right\} \\
= & \frac{2 b \frac{\alpha-2}{2}}{\left(1-\frac{1}{c}\right) B^{3}} \frac{\partial B}{\partial \phi} \\
< & 0 .
\end{aligned}
$$

[16] Y. Chen, S. Zhang, and S. Xu, "Characterizing energy efficiency and deployment efficiency relations for green architecture design," in 2010 IEEE ICC.

[17] W. Wang and G. Shen, "Energy efficiency of heterogeneous cellular network," in Proc. 2010 IEEE VTC - Fall.

[18] K. Tutschku, N. Gerlich, and P. Tran-Gia, "An integrated approach to cellular network planning," in Proc. 1996 International Netw. Planning Symp.

[19] N. Weicker, G. Szabo, K. Weicker, and P. Widmayer, "Evolutionary multiobjective optimization for base station transmitter placement with frequency assignment," IEEE Trans. Evolutionary Computation, vol. 7, no. 2, pp. 189-203, Apr. 2003.

[20] C. Y. Lee and H. G. Kang, "Cell planning with capacity expansion in mobile communications: a tabu search approach," IEEE Trans. Veh. Technol., vol. 49, no. 5, pp. 1678-1690, May 2000.

[21] E. Amaldi, A. Capone, and F. Malucelli, "Planning UMTS base station location: optimization models with power control and algorithms," IEEE Trans. Wireless Commun., vol. 2, no. 4, pp. 939-952, 2003.

[22] E. Amaldi, A. Capone, and F. Malucelli, "Radio planning and coverage optimization of $3 \mathrm{G}$ cellular networks," Wireless Netw., vol. 14, no. 4, pp. 435-447, Aug. 2008.

[23] S. Hanly and R. Mathar, "On the optimal base-station density for CDMA cellular networks," IEEE. Trans. Commun., vol. 50, no. 8, pp. 1274 1281, Aug. 2002.

[24] M. Haenggi, J. G. Andrews, F. Baccelli, O. Dousse, and M Franceschetti, "Stochastic geometry and random graphs for the analysis and design of wireless networks" (invited paper), IEEE J. Sel. Areas Commun., vol. 27, no. 7, pp. 1029-1046, Sept. 2009.

[25] J. G. Andrews, F. Baccelli, and R. K. Ganti, "A tractable approach to coverage and rate in cellular networks," IEEE Trans. Commun., vol. 59 , no. 11, pp. 3122-3134, Nov. 2011.

[26] Y. Zhong and W. Zhang, "Downlink analysis of multi-channel hybrid access two-tier networks," Proc. 2012 IEEE ICC.

[27] S. Singh, H. S. Dhillon, and J. G. Andrews, "Offloading in heterogeneous networks: modeling, analysis and design insights," submitted to IEEE Trans. Wireless Commun. Available: http://arxiv.org/abs/1208. $1977 \mathrm{v} 1$.

[28] B. Rengarajan, G. Rizzo, and M. A. Marsan, "Bounds on QoSconstrained energy savings in cellular access networks with sleep modes," in Proc. 2011 International Teletraffic Congress, pp. 47-54.
[29] R. N. Pupala, L. J. Greenstein, and D. G. Daut, "Throughput analysis of interference-limited MIMO-based cellular systems," IEEE Trans. Wireless Commun., vol. 9, no. 6, pp. 1946-1951, June 2009.

[30] S. Boyd and L. Vandenverghe, Convex Optimization I. Cambridge University Press, 2009.

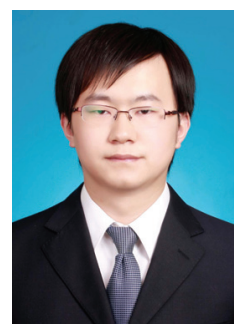

Dongxu Cao (S'10) received his B.S. degree in Electronic Engineering from Tsinghua University, China, in 2011. He is currently a Ph.D. student in Electronic Engineering Department at Tsinghua University. His research interests include network planning and energy-efficient operation for heterogeneous cellular networks.

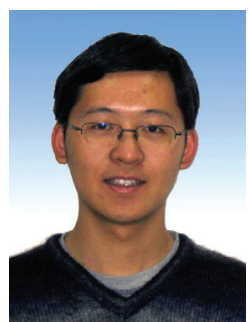

Sheng Zhou (S'06, M'12) received his B.S. and $\mathrm{Ph} . \mathrm{D}$. degrees in Electronic Engineering from Tsinghua University, China, in 2005 and 2011, respectively. $\mathrm{He}$ is now a postdoctoral scholar in Electronic Engineering Department at Tsinghua University, Beijing, China. From January to June 2010, he was a visiting student at Wireless System Lab, Electrical Engineering Department, Stanford University, CA, USA. He is a co-recipient of the Best Paper Award from the 15th Asia-Pacific Conference on Communication (APCC) in 2009, and the 23th IEEE International Conference on Communication Technology (ICCT) in 2011. His research interests include cross-layer design for multiple antenna systems, cooperative transmission in cellular systems, and green wireless cellular communications. 


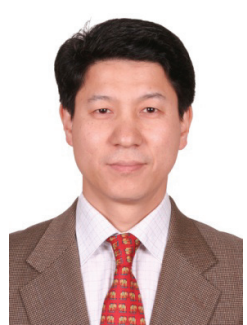

Zhisheng Niu (M'98, SM'99, F'12) graduated from Northern Jiaotong University (currently Beijing Jiaotong University), Beijing, China, in 1985, and got his M.E. and D.E. degrees from Toyohashi University of Technology, Toyohashi, Japan, in 1989 and 1992, respectively. After spending two years at Fujitsu Laboratories Ltd., Kawasaki, Japan, he joined with Tsinghua University, Beijing, China, in 1994, where he is now a professor at the Department of Electronic Engineering and the deputy dean of the School of Information Science and Technology. His major research interests include queueing theory, traffic engineering, mobile Internet, radio resource management of wireless networks, and green communication and networks.

Dr. Niu has been an active volunteer for various academic societies, including council member of Chinese Institute of Electronics (2006-10), vice chair of the Information and Communication Network Committee of Chinese Institute of Communications (2008-12), Councilor of IEICE-Japan (2009-11), and membership development coordinator of IEEE Region 10 (2009-10). In particular, in IEEE Communication Society, he has been serving as an editor of IEEE Wireless Communication Magazine (200912), director of Asia-Pacific Region (2008-09), director for Conference Publications (2010-11), chair of Beijing Chapter (2001-08), and members of Award Committee (2011-13), Emerging Technologies Committee (201012), On-line Content Committee (2010-12), and Strategy Planning Committee. $\mathrm{He}$ has also been serving as general co-chairs of APCC'09/WiCOM'09, TPC co-chairs of APCC'04/ICC'08/WOCC'10/ICCC'12, panel co-chair of WCNC'10, tutorial co-chairs of VTC'10-fall/Globecom'12, and publicity cochairs of PIMRC'10/WCNC'02. He was also the guest co-editors for the IEEE Wireless Communication Magazine Special Issue on Green Radio Communications and Networks (published on Oct. 2011) and the Communication Networks Special Issue on Green Communication Networks (to be published in 2012).

Dr. Niu received the Best Paper Awards from the 13th and 15th Asia-Pacific Conference on Communication (APCC) in 2007 and 2009, respectively, and Outstanding Young Researcher Award from Natural Science Foundation of China in 2009. He is now the fellow of IEEE/IEICE and a distinguished lecturer of IEEE Communication Society (2012-13). 